Answer to Question \#51. Applications of third-order and fifth-order differential equations

H.P.W. Gottlieb

School of Science, Griffith University, Nathan, Queensland 4111, Australia

(October 1999) 


\section{Answer to Question \#51. Applications of third-order and fifth-order differential equations}

Neuenschwander ${ }^{1}$ has asked whether there are any useful applications of thirdorder or fifth-order differential equations. Some Answers have already appeared ${ }^{2,3}$, and subsequently several more applications involving third-order equations were cited and reviewed ${ }^{4}$.

It is well known, as emphasized in an earlier Question from the author ${ }^{5}$, that a third-order nonlinear autonomous differential equation has the lowest order necessary for the possibility of chaotic solutions. Since chaotic phenomena are increasingly being discovered in experimental investigations and are the subject of intense theoretical study, derived third-order equations may eventually be expected to assume greater importance in the description of the physical world.

Some third-order autonomous systems of first-order equations may be rewritten as a third-order differential equation (with respect to time) for one of the dependent variables: this is a jerk equation, as revived in reference 5 and nicely discussed in a broad context by von Baeyer $^{6}$ (see also reference 7).

Alternatively, jerk equations $\dddot{x}=\mathrm{j}(x, \dot{x}, \ddot{x})$ may be studied in their own right. There has been a steady flow of papers in this journal ${ }^{8-10}$ and others ${ }^{11-14}$ as a consequence of the source Question \#38 originally posed by the author in reference 5 concerning the simplest jerk function that may give chaos. Although these equations are in a sense models awaiting an application ${ }^{6}$, some may be physically implemented, for instance as electronic circuits ${ }^{13}$. 
In reference 1, Neuenschwander alluded to the Euler-Lagrange equations of order higher than two. Now, for a Lagrangian whose highest contained derivative is of nth order (say w.r.t. time), the Lagrange's equation then has highest order term ${ }^{15}$ $\left[\mathrm{d}^{\mathrm{n}} / \mathrm{dt}^{\mathrm{n}}\right]\left(\partial \mathrm{L} / \partial \mathrm{x}^{(\mathrm{n})}\right)$ where $(\mathrm{n})$ denotes the $\mathrm{nth}$ order derivative. Provided that $\mathrm{L}$ is not simply linear in $\mathrm{x}^{(\mathrm{n})}$, the highest derivative in the Lagrange's equation will then be of order 2 n, i.e. even. Insofar as many physical phenomena can be expressed by some minimization principle, leading via the calculus of variations to an Euler-Lagrange equation, the scarcity of fundamental physical higher odd-order differential equations may thereby be partly explained.

A further inhibitory factor may come through consideration of self-adjoint operators, desirable in many physical theories because of the reality of eigenvalues. A real differential operator of order $\mathrm{n}$ can be self-adjoint if and only if $\mathrm{n}$ is even ${ }^{16}$ : the highest-order term then has the form ${ }^{16}\left[\mathrm{f}(\mathrm{x}) \mathrm{x}^{(\mathrm{r})}\right]^{(\mathrm{r})}$ with $\mathrm{n}=2 \mathrm{r}$. Actually, unlike a second-order differential equation, a d.e. of even order higher than two cannot always be multiplied by a factor to make it self-adjoint ${ }^{17}$. Thus second-order equations seem to be singled out by this principle, even amongst even-order equations.

As a matter of fact, even the (spatial) fourth-order Euler-Bernoulli beam equation mentioned by Neuenschwander ${ }^{1}$ is obtained from first principles ${ }^{18}$ as a consequence of two (spatial) second-order equations, one for the bending moment (in terms of transverse acceleration) and one for the transverse displacement (in terms of the bending moment), so we are back to basic second-order equations. 
${ }^{1}$ Dwight E. Neuenschwander, "Question \#51. Applications of third-order and fifthorder differential equations," Am. J. Phys. 64 (11), 1353 (1996).

${ }^{2}$ Kirk T. McDonald, "Answer to Question \#51. Applications of third-order and fifthorder differential equations," Am. J. Phys. 66 (4), 277-278 (1998).

${ }^{3}$ George DeRise, "Answer to Question \#51. Applications of third-order and fifth-order differential equations," Am. J. Phys. 66 (4), 278 (1998).

${ }^{4}$ H.P.W. Gottlieb, "Simple nonlinear jerk functions with periodic solutions," Am. J. Phys. 66 (10), 903-906 (1998).

${ }^{5}$ H.P.W. Gottlieb, "Question \#38. What is the simplest jerk function that gives chaos?" Am. J. Phys. 64 (5), 525 (1996).

${ }^{6}$ Hans Christian von Baeyer, "All shook up," The Sciences 38 (1), 12-14 (1998).

${ }^{7}$ Stefan J. Linz, Julien C. Sprott, "The future of chaos," The Sciences 39 (1), 47-48 (1999).

${ }^{8}$ Stefan J. Linz, "Nonlinear dynamical models and jerky motion," Am. J. Phys. 65 (6), 523-526 (1997).

${ }^{9}$ J.C. Sprott, "Some simple chaotic jerk functions," Am. J. Phys. 65 (6), 537-543 (1997).

${ }^{10}$ Stefan J. Linz, "Newtonian jerky dynamics: Some general properties," Am. J. Phys. 66 (12), 1109-1114 (1998).

${ }^{11}$ J.C. Sprott, "Simplest dissipative chaotic flow," Phys. Lett. A 228, 271-274 (1997).

${ }^{12}$ Ralf Eichhorn, Stefan J. Linz and Peter Hanggi, "Transformations of nonlinear dynamical systems to jerky motion and its application to minimal chaotic flows," Phys. Rev. E 58 (6), 7151-7164 (1998).

${ }^{13}$ Stefan J. Linz and J.C. Sprott. "Elementary chaotic flow," Phys. Lett. A 259, 240245 (1999). 
${ }^{14}$ Ralf Eichhorn, Stefan J. Linz and Peter Hanggi, "Classes of dynamical systems being equivalent to a jerky motion," ZAMM 79, S287-S288 (1999).

${ }^{15}$ Lennart Rade and Bertil Westergren, Beta Mathematics Handbook (Studentlitteratur, Sweden, 1990), 2nd ed., p. 317.

${ }^{16}$ Earl A. Coddington and Norman Levinson, Theory of Ordinary Differential Equations (McGraw-Hill, New York, 1955), chap. 7.

${ }^{17}$ Ali Hassan Nayfeh, Introduction to Perturbation Techniques (Wiley, New York, 1981/1993 reprint), p. 434.

${ }^{18}$ Leonard Meirovitch, Elements of Vibration Analysis (McGraw-Hill, New York, 1975), p. 208.

H.P.W. Gottlieb

School of Science

Griffith University

Nathan, Queensland 4111

Australia

h.gottlieb@sct.gu.edu.au 